\title{
Antibiotic Susceptibility of Lactobacillus sp. Isolated from Commercial Probiotic Products by E-Test Strip Method
}

\author{
Poonam Sharma ${ }^{1 *}$, Santosh Anand ${ }^{1}$, Sudhir Kumar Tomar ${ }^{1 *}$ and Pawas Goswami \\ ${ }^{1}$ Dairy Microbiology Division, ICAR-National Dairy Research Institute, Karnal, \\ Haryana, India \\ ${ }^{2}$ Department of Microbiology, Bhaskaracharya College of Applied Sciences, Delhi University, \\ New Delhi, India \\ *Corresponding author
}

\begin{tabular}{|l}
\hline K e y w o r d s \\
Antibiotic, \\
Lactobacillus sp., \\
E-test
\end{tabular}

A B S T R A C T

Minimum Inhibitory Concentration (MIC) of 29 Lactobacillus isolates was determined by E-test strip method according to CLSI and EFSA, 2012 standards. All isolates displayed resistance towards aminoglycosides while $63 \%$ of isolates were found resistant to amikacin (MIC 16-256 $\mu \mathrm{g} / \mathrm{ml}$ ). Strains S8a and S8b (L. rhamnosus and L. acidophilus) were resistant to tetracycline (MIC $256 \mu \mathrm{g} / \mathrm{ml}$ ). Isolates displayed resistance to quinolones [nalidixic acid with MIC $256 \mu \mathrm{g} / \mathrm{ml}$; norfloxacin, ofloxacin, ciprofloxacin and levofloxacin with MIC 6-256 $\mu \mathrm{g} / \mathrm{ml}$; and sparofloxacin (MIC 0.25-4 $\mu \mathrm{g} / \mathrm{ml}$ )], azolidiones [nitrofurantoin (MIC 3-256 $\mu \mathrm{g} / \mathrm{ml}$ )] and cephalosporins (MIC 256 $\mu \mathrm{g} / \mathrm{ml}$ ). Seventy-three percent of isolates displayed resistance against vancomycin while $66 \%$ to teicoplanin. Isolates, S1b (L. reutri), S4 (L. reuteri); S5 (L. plantarum); S8a (L. rhamnosus) and S8b (L. acidophilus) exhibited resistance towards beta-lactams. Very few strains exhibited susceptibility to class macrolides [roxithromycin (S10b- L. plantarum; MIC $0.5 \mu \mathrm{g} / \mathrm{ml}$ ); clarithromycin (S6a- L. plantarum MIC $0.125 \mu \mathrm{g} / \mathrm{ml}$ ); (S12- L. acidophilus MIC 0.25 $\mu \mathrm{g} / \mathrm{ml}$ ) and azithromycin (S12- L. acidophilus MIC $0.75 \mu \mathrm{g} / \mathrm{ml}$ )]. The present study found the prevalence of antibiotic resistance among commercial available probiotics, which may pose a safety risk among humans. Hence, antibiotic sensitivity should be considered as essential for the evaluation of safety assessment of probiotics.

\section{Introduction}

Lactic acid bacteria (LAB) constitute a major group of bacteria, known for their potential health benefits which they impart to the host and have a long history of safe use or generally regarded as safe (GRAS) status. A subset of LAB is generally known as clinically relevant 'probiotic organisms' having health benefits on host. The health benefits of consuming these organisms range from improved intestinal health and immunity, prevention of antibiotic associated diarrhea and even cancer. Probiotic foods are having global popularity and widespread acceptability as depicted by an estimated growth of more than $10 \%$ in last one decade (Wong et al., 2015). Accelerated by increased consumer demand, these health beneficial microorganisms have been comprehensively 
included in a large number of food and pharmaceutical products. Probiotic organisms as pharmaceutical formulations have been prescribed as an adjunct therapy to maintain homeostasis in intestinal microflora, disrupted due to an antibiotic therapy. To re-establish the normal microflora of the intestine, antibiotic resistance in these probiotics is prerequisite, provided of course that they do not pose a threat by way of the transfer of antibiotic resistance genes to other bacteria. The resistance towards antibiotics, if intrinsic, is desirable, however their transfer to pathogenic bacteria offers serious clinical threats. Antibiotic resistance in probiotic organisms is a boon and a ban at the same time. Serious concerns have been raised with this trait of probiotic organisms. A reservoir of antibiotic resistance genes has been established over time because of the extensive use of probiotic organisms in conjunction with antibiotics. Resistance of lactic acid bacteria towards the major groups of antibiotics like beta-lactams, macrolide, aminoglycoside, chloramphenicol and tetracycline has been extensively reviewed (Sharma et al., 2014). The transfer of important antibiotic resistance genes across generations have also been demonstrated among lactobacilli and from lactobacilli to pathogens (Tannock, 1994). These reports have established probiotic bacteria as reservoirs of antibiotic resistant genes that can be transferred to pathogenic strains (Mater, 2008). This raises the question of antibiotic resistances among desired food borne bacteria such as starter and probiotic cultures. The close contact with other bacteria in the human intestine is an excellent pre-condition for horizontal gene transfer with the aid of conjugative transposons and plasmids (Teuber et al., 1999). Therefore, it is very important to validate that probiotic and nutritional LAB strains lack acquired antimicrobial resistance properties prior to considering them safe for human and animal consumption. In the present study, antibiotic resistance of Lactobacillus strains present in market probiotic products, both in pharmaceutical and dairy products in an attempt to contribute to biosafety surveillance of LAB for human consumption was evaluated. The antibiotic resistance of 29 commercial probiotic strains was evaluated by determination of Minimum Inhibitory Concentration (MIC) value using the E-test strip method.

\section{Materials and Methods}

\section{Determination of MIC by E-test strip method}

E-test experiments were performed according to the instruction of the manufacturer and the recommendation of Standard Operating Procedure (SOP) by Kushiro et al., (2009) and Mayerhofer et al., (2008). A total of 30 isolates were isolated from 19 commercial products (Table 1). All isolates were characterized and were subjected to antibiotic susceptibility test using disc diffusion method against a total of 45 antibiotics. Many of them were found to exhibit multiple resistance against commonly used antibiotics. Out of 30 tested isolates, 29 were found to have resistance against a wide array of antibiotics (Sharma et al., 2015). The MIC of 29 probiotic isolates which showed resistance in disc diffusion assay was further tested to determine their MIC values against 30 antibiotics by E-test strip method. List of antibiotics and their concentrations range used in this study are mentioned in Table 2. In brief, single colony of respective isolate was picked from previous streaked De Man Ragosa Sharpe (MRS) agar plate and inoculated in MRS broth for $18 \mathrm{~h}$ at $37^{\circ} \mathrm{C}$. After incubation, cells were harvested by centrifugation $\left(12000 \mathrm{rpm}\right.$ at $4^{\circ} \mathrm{C}$ for $\left.10 \mathrm{~min}\right)$ and resuspended in sterile saline to achieve optical density equivalent to $0.5 \mathrm{McFarland}$ standards (cell density of $10^{8} \mathrm{cfu} / \mathrm{ml}$ ). A 
sterile cotton swab was immersed into the saline suspension, excess fluid was removed, and used to evenly swab the entire surface of the agar plate. One E-test strip containing the particular antibiotic was applied onto the agar using sterile forceps. Plate was inverted and incubated at $37^{\circ} \mathrm{C}$ for $24 \mathrm{~h}$ in an anaerobic jar containing an anaerobic gas pack (Himedia, Mumbai, India), and zone of inhibition (elliptical) was measured. Plates without an antibiotic strip were taken as control plate. The experiments were replicated three times to verify the methodology reproducibility.

\section{Results and Discussion}

According to E-test strip method, MIC of 29 Lactobacillus strains from different commercial probiotic products towards selected antibiotics is summarized in Table 3. Resistance was defined using the previously described breakpoints for Lactobacillus strains (CLSI, 2012; EFSA, 2012). All the strains were found resistant to antibiotics.

The minimal inhibitory concentration is the lowest antibiotic concentration that inhibits the visible bacterial growth after overnight incubation using defined cut-off values for experimentally determined MICs (Philips et al., 1991). Food grade LAB can be categorized as 'susceptible' or 'resistant' to each antibiotic tested (Andrews, 2001). It is considered that when MICs are $\geq 8-16 \mu \mathrm{g} / \mathrm{ml}$ the bacteria may be considered as "moderately resistant"; when MIC is >32 $\mu \mathrm{g} / \mathrm{ml}$ it may be classified as "clinically resistant" to antibiotics. ${ }^{10}$ Results were interpreted and the resistant strains were selected after being compared with known standard given by the CLSI. MIC breakpoints vary considerably depending on the medium and the antibiotics used (Franz et al., 2005). Klare et al., (2005) reported a "general" broth medium for determining LAB antibiotic susceptibilities and showed that this medium, consisting of Iso-Sensitest (90\%) and MRS (10\%) broth, optimally supported the growth of Lactobacillus, Pediococcus, Lactococcus and Bifidobacterium sp. Although, MRS has been used in most studies, as it is suited for the growth of many LAB and their antibiotic susceptibility determinations. In the present study, MIC of the 30 antimicrobial agents against 29 isolates was determined by the Etest strip method using MRS broth. Overall, our results are in good agreement with data from other studies for a broad range of Lactobacillus species and antibiotics (Danielsen and Wind, 2003; Gevers et al., 2003; Perez et al., 2005).

All isolates in our study were found to be sensitive against tetracycline except strain S8a and S8b (L. rhamnosus and L. acidophilus), showed MIC $256 \mu \mathrm{g} / \mathrm{ml}$ towards tetracycline. Similarly, Thumu et al., (2012) determined tetracycline resistance in $L$. plantarum and determined that MIC range was $128 \mu \mathrm{g} / \mathrm{ml}$. The wide range of high MICs obtained was in agreement with a previous study assessing antibiotic susceptibility of 43 L. reuteri strains isolated from piglets (Korhonen et al., 2007).

The Lactobacillus and Bifidobacteria strains showed a wide range of resistance towards streptomycin MICs (2 -256 $\mu \mathrm{g} / \mathrm{ml})$, as reported in some studies (Delgado et al., 2005; Katla et al., 2001). In other studies, Lactobacillus sp. are reported to have a high natural resistance to kanamycin, gentamicin and streptomycin (Blandino et al., 2008; Klare et al., 2007; Nawaz et al., 2011; Zhang et al., 2007). In a study by Hummel et al., (2007) more than $70 \%$ of the isolates were resistant to gentamicin and streptomycin based on the MIC breakpoint values of Scientific Committee on Animal Nutrition (SCAN) and Panel on Additives and Products or Substances used in Animal Feed (FEEDAP) (European Commission, 2002; FEEDAP, 2005). Variability in Lactobacillus 
sp. to these antibiotics has also been evidenced by other authors and tested strains to gentamicin isolated from dairy products and determined that MIC values in gentamicin ranging from 0.06 to $8 \mu \mathrm{g} / \mathrm{ml}$ while resistance to gentamicin (MIC $\geq 16$ $\mathrm{mg} / \mathrm{L})$ was present in strains of $L$. salivarius, L. acidophilus, and L. paracasei (Bujnakova et al., 2014; Hleba et al., 2012; Mayerhofer et al., 2010).

In our study, Lactobacillus strains displayed susceptibility towards gentamicin and tobramycin with MIC in the range 0.19-24 $\mu \mathrm{g} / \mathrm{ml}$ and $(0.19-64 \mu \mathrm{g} / \mathrm{ml})$ respectively, whereas intermediate resistance to kanamycin $(12-256 \mu \mathrm{g} / \mathrm{ml})$ and streptomycin (3-256 $\mu \mathrm{g} / \mathrm{ml}$ ). While more than $58 \%$ of isolates were resistant to kanamycin and $26 \%$ were resistant to streptomycin. Moreover, $63 \%$ of the stains were resistant to amikacin and observed to have MIC in the range $16-256 \mu \mathrm{g} / \mathrm{ml}$ and $23 \%$ of the strains displayed resistance to tobramycin, though only strain S15a showed susceptibility against these antibiotics. Resistance to aminoglycoside antibiotics was previously reported in $\mathrm{LAB}$ and probiotic strains and is considered to be intrinsic in LAB (Charteris et al., 2001; Danielsen and Wind, 2003; Katla et al., 2001). Lower MIC for gentamicin as compared to kanamycin and streptomycin as reported previously and the results was in correlation with our results (Danielsen and Wind, 2003).

In some studies, higher MICs of the aminoglycosides; gentamicin and streptomycin were reported for LAB, which is probably due to the fact that susceptibility testing was performed on MRS agar (E-test) (Charteris et al., 1998). In few studies, streptomycin-resistant Lactobacillus sp. was encountered with MICs of streptomycin $>256 \mathrm{mg} / \mathrm{L}$. The reason for the increased MICs of the aminoglycosides on MRS agar may be due to the medium's low $\mathrm{pH}$ (6.2), because the optimum $\mathrm{pH}$ of the aminoglycosides is in the alkaline range $(\mathrm{pH}$ 7.8) (Amesterdam, 2005).

Some of the isolates in the present study, exhibited complete susceptibility towards macrolides class of antibiotics viz. roxithromycin (S10b- L. plantarum- MIC 0.5 $\mu \mathrm{g} / \mathrm{ml}$ ); clarithromycin (S6a- L. plantarumMIC $0.125 \mu \mathrm{g} / \mathrm{ml}$; S12- L. acidophilus- MIC $0.25 \mu \mathrm{g} / \mathrm{ml}$ ) and azithromycin (S12- $L$. acidophilus- MIC $0.75 \mu \mathrm{g} / \mathrm{ml}$ ). In the current study, class azolidiones (23.33\%) showed resistance towards Lactobacillus sp. All the isolates were found to be highly resistant to nitrofurantoin; displayed MIC in the range (3$256 \mu \mathrm{g} / \mathrm{ml})$.

Vancomycin is considered one of the last antibiotics in the treatment of multidrug resistant pathogens; therefore, its resistance in commercial lactobacilli is of major concern (Bernardeau et al., 2008). Most of the isolated strains in our study were observed to have resistance against vancomycin $(73 \%)$ and teicoplanin $(66 \%)$ except strain S9a, S14a, S14b, S15a, S15b, S16 and S19b for vancomycin and tecioplanin showed susceptibility to these antibiotics leaving S12. Lactic acid bacteria, including $L$. paracasei, L. salivarius, L. plantarum, L. delbrueckii subsp. bulgaricus, L. casei, L. rhamnosus were reported to have mechanisms providing resistance to vancomycin (Aslim and Beyatli, 2004; Comunian et al., 2010; Coppola et al., 2005; Devirgiliis et al., 2011). The resistance of these species to vancomycin is intrinsic, due to the presence of D-Ala-D-lactate in their peptidoglycan instead of the normal dipeptide D-Ala-D-Ala (Ammor et al., 2008). D-ala-D-ala dipeptidase encoded by VanX may act only in the presence of D-ala-D-ala precursor. Intrinsic resistance to vancomycin was earlier also confirmed for L. paracasei, $L$. salivarius and L. plantarum (MIC $\geq 32 \mu \mathrm{g} / \mathrm{L}$ ) (Blandino et al., 2008). 
Table.1 Commercial probiotic dairy and pharmaceutical products with organisms mentioned on the products and their origin

\begin{tabular}{|c|c|c|c|}
\hline S. No. & $\begin{array}{l}\text { Probiotic capsules and } \\
\text { dairy products }\end{array}$ & Strains isolated & Origin \\
\hline 1 & S1 & L. rhamnosus, L. reuteri & Pharmaceutical product \\
\hline 2 & S2 & L. casei & Dairy product \\
\hline 3 & S3 & L. plantarum & Pharmaceutical product \\
\hline 4 & S4 & L. reuteri & Pharmaceutical product \\
\hline 5 & S5 & L. plantarum & Pharmaceutical product \\
\hline 6 & S6 & L. plantarum, L. rhamnosus & Pharmaceutical product \\
\hline 7 & S7 & L. plantarum, L. rhamnosus & Pharmaceutical product \\
\hline 8 & S8 & L. rhamnosus, L. acidophilus & Pharmaceutical product \\
\hline 9 & S9 & L. rhamnosus, L. acidophilus & Pharmaceutical product \\
\hline 10 & S10 & L. fermentum,, L. plantarum & Dairy product \\
\hline 11 & S11 & L. acidophilus, L. plantarum & Dairy product \\
\hline 12 & S12 & L. acidophilus & Pharmaceutical product \\
\hline 13 & S13 & L. acidophilus, L. plantarum & Dairy product \\
\hline 14 & S14 & L. acidophilus, L. plantarum & Dairy product \\
\hline 15 & S15 & L. acidophilus, L. plantarum & Dairy product \\
\hline 16 & S16 & L. rhamnosus & Pharmaceutical product \\
\hline 17 & S17 & L. rhamnosus & Pharmaceutical product \\
\hline 18 & S18 & L. rhamnosus & Pharmaceutical product \\
\hline 19 & S19 & L. rhamnosus, L. acidophilus & Pharmaceutical product \\
\hline
\end{tabular}

Table.2 List of antibiotics and their range of concentration used for determination of MIC

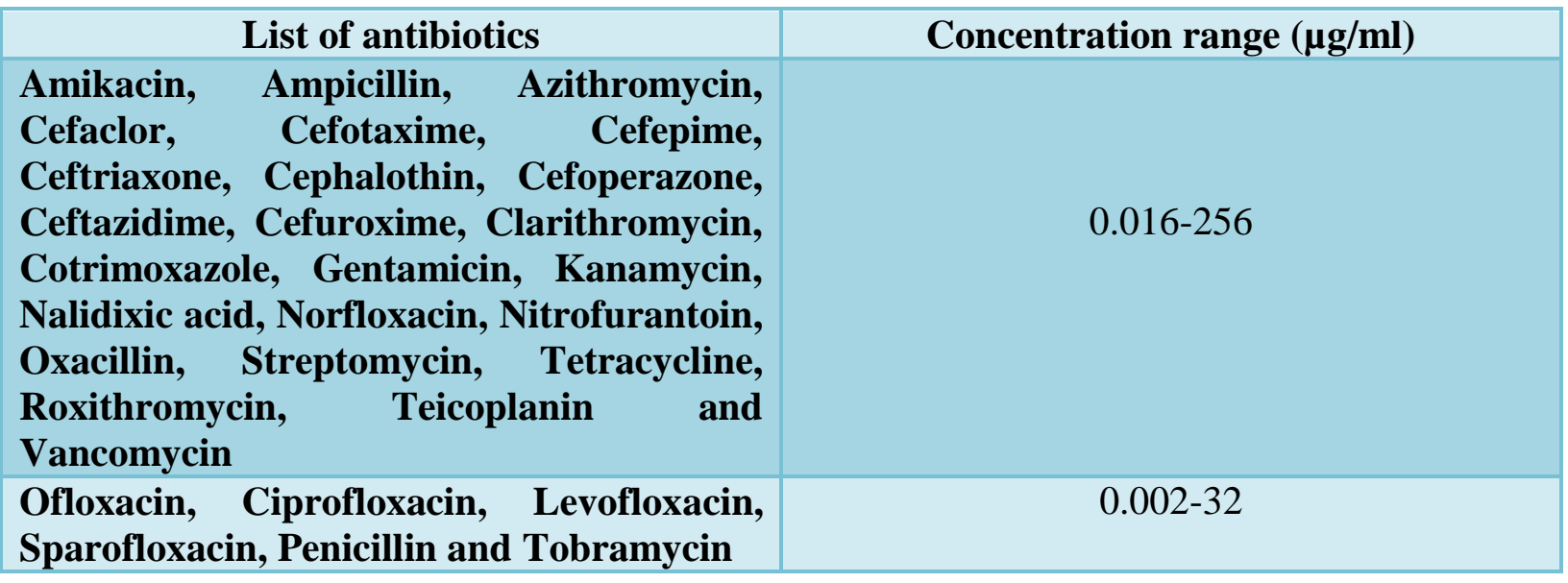


Table.3 Susceptibility of 29 Lactobacillus strains to selected antibiotics as determined by the E-test method using MRS medium

\begin{tabular}{|c|c|c|c|c|c|c|c|c|c|c|}
\hline \multicolumn{11}{|c|}{ Commercial products with bacterial strains } \\
\hline & & & $\begin{array}{c}\text { (S1a) } \\
\text { L. } \\
\text { rhamnosus }\end{array}$ & $\begin{array}{l}(\mathrm{S} 1 \mathrm{~b}) \\
L . \\
\text { reuteri }\end{array}$ & $\begin{array}{c}(\mathrm{S} 2) \\
L . \\
\text { casei }\end{array}$ & $\begin{array}{c}(\mathrm{S} 3) \\
L . \\
\text { plantarum }\end{array}$ & $\begin{array}{c}(\mathrm{S} 4) \\
L . \\
\text { reuteri }\end{array}$ & $\begin{array}{c}\text { (S5) } \\
\text { L. } \\
\text { plantarum }\end{array}$ & $\begin{array}{c}\text { (S6a) } \\
\text { L. } \\
\text { plantarum }\end{array}$ & $\begin{array}{c}\text { (S6b) } \\
\text { L. } \\
\text { rhamnosus }\end{array}$ \\
\hline $\begin{array}{c}\text { Different Classes } \\
\text { of Antibiotics }\end{array}$ & Antibiotics & $\begin{array}{l}\text { MIC range } \\
(\mu \mathrm{g} / \mathrm{ml})\end{array}$ & \multicolumn{8}{|c|}{ Zone of Inhibition } \\
\hline \multirow[t]{5}{*}{ Aminoglycosides } & Amikacin & $0.016-256$ & 64 & 16 & 48 & 256 & 48 & 256 & 32 & 96 \\
\hline & Tobramycin & $0.016-256$ & - & 6 & - & - & 12 & 32 & - & 24 \\
\hline & Gentamicin & $0.016-256$ & 6 & 2 & - & 12 & - & 6 & 6 & 4 \\
\hline & Kanamycin & $0.016-256$ & 64 & 12 & - & 256 & 24 & 256 & 32 & 32 \\
\hline & Streptomycin & $0.016-256$ & 24 & 12 & 48 & 12 & 12 & 4 & 64 & 24 \\
\hline Macrolides & Clarithromycin & $0.016-256$ & - & - & - & - & - & - & .125 & - \\
\hline Azolidiones & Nitrofurantoin & $0.016-256$ & - & 32 & 48 & - & 48 & 4 & 24 & - \\
\hline \multirow[t]{2}{*}{ Glycopeptides } & Teicoplanin & $0.016-256$ & 256 & 256 & 256 & 256 & 256 & 256 & 256 & 256 \\
\hline & Vancomycin & $0.016-256$ & 256 & 256 & 256 & 256 & 256 & 256 & 256 & 256 \\
\hline \multirow[t]{3}{*}{ Beta lactams } & Ampicillin & $0.016-256$ & 1.5 & 16 & - & .75 & 256 & - & - & - \\
\hline & Penicillin & $0.002-32$ & - & 256 & - & - & 256 & 256 & - & - \\
\hline & Oxacillin & $0.016-256$ & - & 256 & - & - & 256 & - & - & - \\
\hline
\end{tabular}


Int.J.Curr.Microbiol.App.Sci (2018) 7(4): 3499-3517

\begin{tabular}{|c|c|c|c|c|c|c|c|c|c|c|}
\hline \multirow[t]{7}{*}{ Cephalosporins } & Cefaclor & $0.016-256$ & 48 & 256 & 32 & - & - & 256 & - & - \\
\hline & Cephalothin & $0.016-256$ & - & - & - & - & 256 & 1 & - & - \\
\hline & Ceftazidime & $0.002-32$ & 32 & 6 & - & - & - & 1 & - & - \\
\hline & Cefepime & $0.016-256$ & 256 & 4 & 256 & - & 6 & .64 & 256 & - \\
\hline & Ceftriaxone & $0.016-256$ & 64 & 2 & - & - & - & .094 & - & - \\
\hline & Cefuroxime & $0.016-256$ & - & - & - & - & - & .75 & - & - \\
\hline & Cefoperazone & $0.016-256$ & - & - & - & - & - & 32 & - & - \\
\hline \multirow[t]{6}{*}{ Quinolones } & Norfloxacin & $0.016-256$ & - & 256 & - & 256 & 256 & 256 & - & 256 \\
\hline & Ofloxacin & $0.002-32$ & - & - & - & 256 & - & 256 & - & - \\
\hline & Ciprofloxacin & $0.002-32$ & - & 256 & - & - & 256 & 256 & - & 256 \\
\hline & Nalidixic Acid & $0.016-256$ & 256 & 256 & 256 & 256 & 256 & 256 & 256 & 256 \\
\hline & Levofloxacin & $0.002-32$ & - & - & - & - & - & 6 & - & - \\
\hline & Sparofloxacin & $0.002-32$ & - & - & - & - & - & 4 & - & - \\
\hline Sulfonamides & $\begin{array}{c}\text { Co- } \\
\text { Trimoxazole }\end{array}$ & $0.016-256$ & 256 & 256 & 256 & - & 256 & - & 256 & 256 \\
\hline
\end{tabular}




\begin{tabular}{|c|c|c|c|c|c|c|c|c|c|}
\hline \multicolumn{10}{|c|}{ Commercial products with bacterial strains } \\
\hline & & & $\begin{array}{c}\text { (S7a) } \\
\text { L. } \\
\text { rhamnosus }\end{array}$ & $\begin{array}{c}\text { (S7b) } \\
\text { L. } \\
\text { plantarum }\end{array}$ & $\begin{array}{c}\text { (S8a) } \\
\text { L. } \\
\text { rhamnosus }\end{array}$ & $\begin{array}{c}(\mathrm{S} 8 \mathrm{~b}) \\
\text { L. } \\
\text { acidophilus }\end{array}$ & $\begin{array}{c}\text { (S9) } \\
\text { L. } \\
\text { rhamnosus }\end{array}$ & $\begin{array}{c}\text { (S10a) } \\
L . \\
\text { fermentum }\end{array}$ & $\begin{array}{c}\text { (S10b) } \\
\text { L. } \\
\text { plantarum }\end{array}$ \\
\hline $\begin{array}{l}\text { Different Classes } \\
\text { of Antibiotics }\end{array}$ & Antibiotics & $\begin{array}{l}\text { MIC range } \\
(\mu \mathrm{g} / \mathrm{ml})\end{array}$ & \multicolumn{7}{|c|}{ Zone of Inhibition $(\mu \mathrm{g} / \mathrm{ml})$} \\
\hline Tetracycline & Tetracycline & $0.016-256$ & - & - & 256 & 256 & - & - & - \\
\hline \multirow{5}{*}{ Aminoglycosides } & Tobramycin & $0.016-256$ & 32 & 64 & 32 & 16 & - & - & 12 \\
\hline & Gentamicin & $0.016-256$ & 4 & 8 & 24 & 16 & - & - & - \\
\hline & Kanamycin & $0.016-256$ & 256 & 64 & 256 & 256 & - & 32 & 16 \\
\hline & Amikacin & $0.016-256$ & 96 & 48 & 256 & 256 & - & 16 & 16 \\
\hline & Streptomycin & $0.016-256$ & 256 & 48 & 48 & 24 & 4 & - & 24 \\
\hline Macrolides & Roxithromycin & $0.016-256$ & - & - & - & - & - & - & .5 \\
\hline Azolidiones & Nitrofurantoin & $0.016-256$ & 256 & - & 256 & 256 & - & - & - \\
\hline \multirow[b]{2}{*}{ Glycopeptides } & Vancomycin & $0.016-256$ & 256 & 256 & 256 & 256 & - & 256 & 256 \\
\hline & Teicoplanin & $0.016-256$ & 256 & 256 & 256 & 256 & - & 256 & 256 \\
\hline \multirow[b]{2}{*}{ Beta lactams } & Ampicillin & $0.016-256$ & - & 2 & 24 & 24 & .75 & - & - \\
\hline & Penicillin & $0.002-32$ & - & - & 16 & 256 & - & - & - \\
\hline
\end{tabular}


Int.J.Curr.Microbiol.App.Sci (2018) 7(4): 3499-3517

\begin{tabular}{|c|c|c|c|c|c|c|c|c|c|}
\hline & Amoxycillin & $0.016-256$ & - & - & - & 3 & - & - & - \\
\hline \multirow{8}{*}{ Cephalosporins } & Cefaclor & $0.016-256$ & 256 & 48 & 256 & 256 & - & 64 & 32 \\
\hline & Cefotaxime & $0.016-256$ & - & - & 256 & 256 & - & - & - \\
\hline & Cephalothin & $0.016-256$ & - & - & - & 256 & - & - & - \\
\hline & Cefoperazone & $0.016-256$ & - & - & 256 & 256 & - & - & - \\
\hline & Ceftazidime & $0.016-256$ & - & 16 & 256 & 256 & 256 & 8 & 6 \\
\hline & Cefepime & $0.016-256$ & 256 & 256 & 256 & 256 & 256 & - & - \\
\hline & Ceftriaxone & $0.016-256$ & 256 & 48 & 256 & 256 & - & - & 8 \\
\hline & Cefuroxime & $0.016-256$ & 4 & 2 & - & 256 & - & - & - \\
\hline \multirow{3}{*}{ Quinolones } & Norfloxacin & $0.016-256$ & - & - & 8 & 4 & - & 24 & 24 \\
\hline & Ofloxacin & $0.002-32$ & - & - & - & - & - & - & 12 \\
\hline & Nalidixic Acid & $0.016-256$ & 256 & 256 & 256 & 256 & 256 & 256 & 256 \\
\hline Sulfonamides & Cotrimoxazole & $0.016-256$ & 256 & 256 & 256 & 256 & .38 & 256 & 1 \\
\hline
\end{tabular}




\begin{tabular}{|c|c|c|c|c|c|c|c|c|c|c|}
\hline \multicolumn{11}{|c|}{ Commercial products with bacterial strains } \\
\hline & & & $\begin{array}{c}\text { (S11a) } \\
\text { L. } \\
\text { acidophilus }\end{array}$ & $\begin{array}{c}(\mathrm{S} 11 \mathrm{~b}) \\
L . \\
\text { plantarum }\end{array}$ & $\begin{array}{c}\text { (S12) } \\
\text { L. } \\
\text { acidophilus }\end{array}$ & $\begin{array}{c}\text { (S13a) } \\
\text { L. } \\
\text { acidophilus }\end{array}$ & $\begin{array}{c}\text { (S13b) } \\
L . \\
\text { plantarum }\end{array}$ & $\begin{array}{c}\text { (S14a) } \\
\text { L. } \\
\text { plantarum }\end{array}$ & $\begin{array}{c}\text { (S14b) } \\
\text { L. } \\
\text { acidophilus }\end{array}$ & $\begin{array}{c}\text { (S15a) } \\
\text { L. } \\
\text { plantarum }\end{array}$ \\
\hline $\begin{array}{c}\text { Different } \\
\text { Classes } \\
\text { of Antibiotics }\end{array}$ & Antibiotics & $\begin{array}{l}\mathrm{MIC} \\
\text { range } \\
(\mu \mathrm{g} / \mathrm{ml})\end{array}$ & \multicolumn{8}{|c|}{ Zone of Inhibition $(\mu \mathrm{g} / \mathrm{ml})$} \\
\hline \multirow{5}{*}{$\begin{array}{l}\text { Amino- } \\
\text { glycosides }\end{array}$} & Gentamicin & $0.016-256$ & 2 & 3 & 6 & - & - & 8 & - & .19 \\
\hline & Amikacin & $0.016-256$ & 24 & 16 & 256 & 24 & 32 & 32 & 64 & .5 \\
\hline & Tobramycin & $0.002-32$ & - & 12 & - & 32 & - & 64 & 24 & .19 \\
\hline & Kanamycin & $0.016-256$ & 32 & 32 & 256 & 48 & 24 & - & - & - \\
\hline & Streptomycin & $0.016-256$ & 12 & 16 & 256 & 32 & 24 & 16 & 3 & - \\
\hline & Clarithromycin & $0.016-256$ & - & - & .25 & - & - & - & - & - \\
\hline Macrolides & Azithromycin & $0.016-256$ & - & - & .75 & - & - & - & - & - \\
\hline Azolidiones & Nitrofurantoin & $0.016-256$ & - & - & - & - & 4 & - & 256 & 4 \\
\hline & Vancomycin & $0.016-256$ & 256 & 256 & 256 & 256 & 256 & - & - & - \\
\hline Glycopeptides & Teicoplanin & $0.016-256$ & 256 & 256 & - & 256 & .75 & - & - & - \\
\hline Beta lactams & Ampicillin & $0.016-256$ & - & - & - & - & - & .19 & .75 & - \\
\hline Cephalosporins & Cefepime & $0.016-256$ & - & - & - & - & - & - & - & 256 \\
\hline
\end{tabular}


Int.J.Curr.Microbiol.App.Sci (2018) 7(4): 3499-3517

\begin{tabular}{|c|c|c|c|c|c|c|c|c|c|c|}
\hline & Norfloxacin & $0.016-256$ & - & 12 & 256 & 256 & 32 & 24 & - & 2 \\
\hline \multirow{5}{*}{ Quinolones } & Ofloxacin & $0.016-256$ & - & 6 & 256 & - & - & - & - & - \\
\hline & Ciprofloxacin & $0.002-32$ & - & 3 & - & - & - & - & 256 & .25 \\
\hline & Nalidixic Acid & $0.016-256$ & 256 & 256 & 256 & 256 & 256 & 256 & 256 & 256 \\
\hline & Levofloxacin & $0.002-32$ & - & - & - & - & - & - & 256 & - \\
\hline & Sparofloxacin & $0.002-32$ & - & - & - & - & - & 3 & .25 & - \\
\hline Sulfonamides & Cotrimoxazole & $0.016-256$ & 256 & 256 & - & - & 256 & 256 & 2 & .19 \\
\hline
\end{tabular}




\begin{tabular}{|c|c|c|c|c|c|c|c|c|}
\hline \multicolumn{9}{|c|}{ Commercial products with bacterial strains } \\
\hline & & & $\begin{array}{l}(\mathrm{S} 15 \mathrm{~b}) \\
\text { L. acidophilus }\end{array}$ & $\begin{array}{l}(\mathrm{S} 16) \\
\text { L. rhamnosus }\end{array}$ & $\begin{array}{l}(\mathrm{S} 17) \\
\text { L. rhamnosus }\end{array}$ & $\begin{array}{l}(\mathrm{S} 18) \\
\text { L. rhamnosus }\end{array}$ & $\begin{array}{l}(\mathrm{S} 19 \mathrm{a}) \\
\text { L. rhamnosus }\end{array}$ & $\begin{array}{c}(\mathrm{S} 19 \mathrm{~b}) \\
\text { L. acidophilus }\end{array}$ \\
\hline $\begin{array}{l}\text { Different Classes } \\
\text { of Antibiotics }\end{array}$ & Antibiotics & $\begin{array}{c}\text { Minimum } \\
\text { Inhibitory } \\
\text { Concentration } \\
\text { (MIC) } \mu \mathrm{g} / \mathrm{ml}\end{array}$ & \multicolumn{6}{|c|}{ Zone of Inhibition $(\mu \mathrm{g} / \mathrm{ml})$} \\
\hline \multirow{5}{*}{ Aminoglycosides } & Gentamicin & $0.016-256$ & 12 & - & 12 & 4 & - & - \\
\hline & Amikacin & $0.016-256$ & 64 & - & 256 & 32 & 256 & - \\
\hline & Tobramycin & $0.016-256$ & 32 & - & 24 & 8 & - & - \\
\hline & Kanamycin & $0.016-256$ & 256 & - & - & 32 & 24 & - \\
\hline & Streptomycin & $0.016-256$ & 12 & - & - & 16 & 32 & - \\
\hline Azolidiones & Nitrofurantoin & $0.016-256$ & 16 & 4 & 12 & 3 & - & - \\
\hline \multirow[b]{2}{*}{ Glycopeptides } & Vancomycin & $0.016-256$ & - & - & 256 & 256 & 256 & - \\
\hline & Teicoplanin & $0.016-256$ & - & - & 256 & 256 & 256 & - \\
\hline \multirow{4}{*}{ Cephalosporins } & Ceftazidime & $0.002-32$ & - & - & 32 & 16 & - & - \\
\hline & Cefaclor & $0.016-256$ & 16 & - & - & 32 & - & - \\
\hline & Cefepime & $0.016-256$ & 2 & 2 & - & 256 & - & .75 \\
\hline & Ceftriaxone & $0.016-256$ & - & - & - & 256 & - & - \\
\hline \multirow[b]{4}{*}{ Quinolones } & Norfloxacin & $0.016-256$ & 32 & - & 4 & 4 & 256 & - \\
\hline & Ofloxacin & $0.002-32$ & - & - & - & - & 256 & - \\
\hline & Ciprofloxacin & $0.016-256$ & 256 & - & - & - & 256 & - \\
\hline & $\begin{array}{l}\text { Nalidixic } \\
\text { Acid }\end{array}$ & $0.016-256$ & 256 & - & - & - & 256 & 256 \\
\hline Sulfonamides & $\begin{array}{l}\text { Cotrimox- } \\
\text { azole }\end{array}$ & $0.016-256$ & 8 & - & 256 & 256 & - & - \\
\hline
\end{tabular}


The cell wall impermeability seems to have mechanism of resistance to beta-lactam antibiotics (Condon, 1983). Furthermore, the cooperation of non-specific mechanisms, such as multi-drug transporters (Putman et al., 2001) and defective cell wall autolytic systems (Kim et al., 1982) may also account for differences between strains within the same species. The strains tested in this study showed the resistance towards cell wall synthesis inhibitors (beta-lactams) while strains named S1b and S4 (L. reuteri), S5 (L. plantarum), S8a (L. rhamnosus) and S8b (L. acidophilus) displayed resistance to ampicillin, penicillin and oxacillin with MIC in the range (MIC 16-256 $\mu \mathrm{g} / \mathrm{ml}$ ) while the rest of the strains were found to be sensitive against these antibiotics. Strains S1b and S4 (L. reuteri), S5 ( $L$. plantarum), S8a ( $L$. rhamnosus) and $\mathrm{S} 8 \mathrm{~b}$ (L. acidophilus) were resistant to penicillin (MIC 16-256 $\mu \mathrm{g} / \mathrm{ml}$ ). While for oxacillin two strains were resistant (S1b and S4) and displayed MIC $256 \mu \mathrm{g} / \mathrm{ml}$, although low resistance towards this antibiotic has also been observed among other strains of Lactobacillus sp. and for amoxicillin strain $\mathrm{S} 8 \mathrm{~b}$ was found to be susceptible and exhibited MIC $3 \mu \mathrm{g} / \mathrm{ml}$. On the other hand, a widespread resistance to penicillins, especially penicillin $\mathrm{G}$, has already been observed in lactobacilli used as probiotics or starter cultures in L. rhamnosus, L. reuteri and L. plantarum isolated from cheese (Flórez et al., 2005; Čanžek Majhenič et al., 2007; Belleti et al., 2009), in L. delbrueckii subsp. bulgaricus from Chinese yogurts (Zhou et al., 2012), in L. casei from fermented milk "Dahi" (Soomro and Masud, 2012), in L. casei, L. helveticus and L. plantarum from fermented milk and vegetables (Lapsiri et al., 2011; Yüksekdağ and Beyatli, 2008), in $L$. salivarius, L. curvatus and L. sakei from different fermented foods and beverages (Gevers et al., 2003; Nawaz et al., 2011). While other studies showed that certain ampicillin-resistant lactobacilli were also isolated from Nigerian fermented foods and beverages (Olukoya et al., 1993) as well as fermented milk in India (Lavanya et al., 2011; Soomro and Masud, 2012). Resistance to oxacillin was also shown to occur in lactobacilli (especially L. rhamnosus) isolated from dairy products such as different types of cheeses (Coppola et al., 2005; Herreros et al., 2005; Hummel et al., 2007).

Lactobacillus strains exhibited MIC in the range (16-256 $\mu \mathrm{g} / \mathrm{ml})$ against cefaclor, followed by cephalothin and ceftazidime (1$256 \mu \mathrm{g} / \mathrm{ml})$, cefepime (4-256 $\mu \mathrm{g} / \mathrm{ml})$, ceftriaxone $(0.094-256 \mu \mathrm{g} / \mathrm{ml})$, and for cefuroxime $(0.75-256 \mu \mathrm{g} / \mathrm{ml})$. Generally, Lactobacillus species were found more resistant to cephalosporins; cephalothin, cefuroxime, ceftriaxone and cefoxitin (Abriouel et al., 2015). The resistance mechanism is not fully elaborated, but cell wall impermeability and non-specific multidrug transporters may be involved (Ammor et al., 2007). Resistance to these compounds was also shown in L. paracasei from yogurts (Honi et al., 2013), in $L$. plantarum and $L$. pentosus from fermented olives (Casado et al., 2014) and in L. curvatus and $L$. fermentum from fermented sausages (Zdolec et al., 2011).

Lactobacilli seem to be intrinsically resistant to quinolones, e.g. ciprofloxacin and nalidixic acid, by unknown resistance mechanism. The intrinsic resistance to ciprofloxacin was not found to be associated with mutations in the QRDR of gyrA and parC as occurred in grampositive bacteria, thus the intrinsic resistance could have resulted from intrinsic characteristics such as cell wall structure, permeability, or an efflux mechanism (Hummel et al., 2007). In our study, all the strains were found to be completely resistant towards nalidixic acid (nearly 86\%) and found to have MIC $256 \mu \mathrm{g} / \mathrm{ml}$. Resistance towards norfloxacin has been observed in 
almost all strains displayed (MIC 4-256 $\mu \mathrm{g} / \mathrm{ml}$ ), followed by ofloxacin (S3, S5, S10b, $\mathrm{S} 11 \mathrm{~b}, \mathrm{~S} 12$ and S19a; MIC 6-256 $\mu \mathrm{g} / \mathrm{ml}$ ), ciprofloxacin (S1b, S5, S4, S6b, S11b, S15b and S19a; MIC 0.25-256 $\mu \mathrm{g} / \mathrm{ml}$ ), levofloxacin (S14b and S15b; MIC 6 and $256 \mu \mathrm{g} / \mathrm{ml}$ ) and sparofloxacin (S14a and S14b; MIC 0.25-4 $\mu \mathrm{g} / \mathrm{ml}$ ) and strain S5 exhibited susceptibility to levofloxacin and sparofloxacin. While ciprofloxacin resistance was reported for more than $60 \%$ of the LAB strains examined. A total of $60-77.8 \%$ of starter and probiotic strains of LAB was resistant to ciprofloxacin (Hummel et al., 2007; Zarazaga et al., 1999).

In the current study, class sulfonamides $(56.66 \%)$ showed resistance towards Lactobacillus sp. All the isolates were found to be highly resistant towards cotrimoxazole (MIC $0.38-256 \mu \mathrm{g} / \mathrm{ml}$ ). Resistance to other inhibitors of nucleic acid synthesis such as trimethoprim and sulfonamides has also been reported as an intrinsic feature (Katla et al., 2001). In some cases, sulfamethoxazole/ trimethoprim phenotypic determination of susceptibility of lactobacilli in some culture media may not be coherent, because certain antagonistic medium components such as paminobenzoic acid (PABA) and thymidine may interfere with the antibiotic activity (Turnidge and Bell, 2005). In other cases, the mechanisms of resistance in lactobacilli isolated from fermented foods include cell wall impermeability, alternative metabolic pathways, a dihydrofolate reductase (DHFR), that is insensitive to trimethoprim, overproduction of DHFR and trimethopriminsensitive transferable DHFRs (Huovinen, 1987).

A number of researchers examined antibiotic resistance of bacteria isolated from different food samples have argued that the results of antibiotic resistance vary from study to study (Lira et al., 2004; Picozzi et al., 2005; Caro et al., 2007). It was concluded that this phenomenon may be due to high spontaneous frequency of mutation to antibiotic resistance which is not uncommon in lactobacilli (Curragh and Collins, 1992). Additionally, the loss of antibacterial activity of unstable antimicrobial agents during incubation may result in sub inhibitory concentrations that could promote the emergence of resistant strains during prolonged exposure (Herra et al., 1995).

Nevertheless, broth micro dilution provides a simple method to determine MICs for a large number of strains and antibiotics, whereas the E-test could be more suitable for testing single strains. However, resistant and susceptible strains were generally more clearly separated by E-test in the present investigation due to the wider and more precise antibiotic concentration range of the E-test. These findings reinforce the antimicrobial susceptibility testing in the safety assessment procedure of strains intended for probiotic or nutritional use especially in humans. All the isolates were having MIC higher than the value prescribed by CLSI against a number of antibiotics. The results have indicated the presence of multiple drug resistance is in most of the isolates in different species of probiotic strains, which is detrimental to food safety.

\section{Acknowledgments}

The authors thank the Director of ICARNDRI for supporting the work.

\section{References}

Abriouel, H., Muñoz, M. D. C. C., Lerma, L. L., Montoro, B. P., Bockelmann, W., Pichner, R., Kabisch, J., Cho, G. S., Franz, C. M., Gálvez, A., and Benomar, N. 2015. New insights in antibiotic resistance of Lactobacillus species from 
fermented foods. Food Research International, 78: 465-481.

Ammor, M. S., Flórez, A. B., Alvarez-Martín, P., Margolles, A., and Mayo, B. 2008. Analysis of tetracycline resistance tet $(W)$ genes and their flanking sequences in intestinal Bifidobacterium species. Journal of Antimicrobial Chemotherapy, 62: 688-693.

Ammor, M. S., Florez, A. B., and Mayo, B. 2007. Antibiotic resistance in nonenterococcal lactic acid bacteria and bifidobacteria. Food Microbiology, 24: 559-570.

Amsterdam, D. 2005. Susceptibility testing of antimicrobials in liquid media. In: Lorian V, editor. Antibiotics in Laboratory Medicine. Baltimore: Williams and Wilkins, 61-143.

Andrews, M. J. 2001. Determination of minimum inhibitory concentrations. Journal of Antimicrobial Chemotherapy, 48: 5-16.

Aslim, B., and Beyatli, Y. 2004. Antibiotic resistance and plasmid DNA contents of $S$. thermophilus strains isolated from Turkish yoghurts. Turkish Journal of Veterinary and Animal Sciences, 28: 257-263.

Belletti, N., Gatti, M., Bottari, B., Neviani, E., Tabanelli, G., and Gardini, F. 2009. Antibiotic resistance of lactobacilli isolated from two Italian hard cheeses. Journal of Food Protection, 72: 21622169.

Bernardeau, M., Vernoux, J. P., HenriDubernet, S., and Guéguen, M. 2008. Safety assessment of dairy microorganisms: The Lactobacillus genus. International Journal of Food Microbiology, 126: 278-285.

Blandino, G., Milazzo, I., and Fazio, D. 2008. Antibiotic susceptibility of bacterial isolates from probiotic products available in Italy. Microbial Ecology in Health and Disease, 20: 199-203.
Bujnakova, D., Strakova, E., and Kmet, V. 2014. In vitro evaluation of the safety and probiotic properties of Lactobacilli isolated from chicken and calves. Anaerobe, 29: 118-127.

Canzek, Majhenic. A., Mohar, Lorbeg, P., and Rogelj, I. 2007. Characterization of the Lactobacillus community in traditional Karst ewe's cheese. International Journal of Dairy Technology, 60: 182190.

Caro, I., Mateo, J., and Garcia-Armesto, M. R. 2007. Phenotypical characteristics of Shiga-like toxin Escherichia coli isolated from sheep dairy products. Letters in Applied Microbiology, 45: 295-300.

Casado-Muñoz, M. C., Benomar, N., LavillaLerma, L., Gálvez, A., and Abriouel, H. 2014. Antibiotic resistance of Lactobacillus pentosus and Leuconostoc pseudomesenteroides isolated from naturally-fermented Aloreña table olives throughout fermentation process. International Journal of Food Microbiology, 172: 110-118.

Charteris, W. P., Kelly, P. M., Morelli, L., and Collins, J. K. (2001). Gradient diffusion antibiotic susceptibility testing of potentially probiotic lactobacilli. Journal of Food Protection, 64: 20072014.

Charteris, W. P., Kelly, P. M., Morelli, L., and Collins, J. K. 1998. Development and application of an in vitro methodology to determine the transit tolerance of potentially probiotic Lactobacillus and Bifidobacterium species in the upper human gastrointestinal tract. Journal of Applied Microbiology, 84: 759-768.

Clinical and Laboratory Standards Institute. 2012. Performance Standards for Antimicrobial Susceptibility Testing: Twenty- Second Informational Supplement. CLSI Document M100- 
S22, Clinical Laboratory Standard Institute, Wayne, PA.

Comunian, R., Daga, E., Dupré, I., Paba, A., Devirgiliis, C., Piccioni, V., Perozzi, G., Zonenschain, D., Rebecchi, A., Morelli, L., Lorentiis, A., and Giraffa, G. 2010. Susceptibility to tetracycline and erythromycin of Lactobacillus paracasei strains isolated from traditional Italian fermented foods. International Journal of Food Microbiology, 138: 151-156.

Condon, S. 1983. Aerobic metabolism of lactic acid bacteria. Irish Journal of Food Science and Technology, 7, 1525.

Coppola, R., Succi, M., Tremonte, P., Reale, A., Salzano, G., and Sorrentino, E. 2005. Antibiotic susceptibility of Lactobacillus rhamnosus strains isolated from Parmigiano Reggiano cheese. Le Lait, 85: 193-204.

Curragh, H. J., and Collins, M. A. 1992. High-levels of spontaneous drugresistance in Lactobacillus. Journal of Applied Bacteriology, 73: 31-36.

Danielsen, M., and Wind, A. A. 2003. Susceptibility of Lactobacillus spp. to antimicrobial agents. International Journal of Food Microbiology, 82: 111.

Delgado, S., Florez, A. B., and Mayo, B. 2005. Antibiotic susceptibility of Lactobacillus and Bifidobacterium species from human gastro-intestinal tract. Current Microbiology, 50: 202 207.

Devirgiliis, C., Barile, S., and Perozzi, G. 2011. Antibiotic resistance determinants in the interplay between food and gut microbiota. Genes and Nutrition, 6: 275-284.

European Commission. 2002. Opinion of the Scientific Committee on Animal Nutrition on the criteria for assessing the safety of micro-organisms resistant to antibiotics of human clinical and veterinary importance. European Commission, Health and Consumer Protection Directorate General, Directorate C, Scientific Opinions, Brussels, Belgium.

European Food Safety Authority (2012). Scientific opinion on the maintenance of the list of QPS biological agents intentionally added to food and feed. EFSA Journal, 10: 1-84.

FEEDAP Panel. 2005. Opinion of the Scientific Panel on Additives and Products or Substances Used in Animal Feed on the updating of the criteria used in assessment of bacteria for resistance to antibiotics of human and veterinary importance. EFSA, 223: 1-12.

Florez, A. B., Delgado, S., and Mayo, B. 2005. Antimicrobial susceptibility of lactic acid bacteria isolated from a cheese environment. Canadian Journal of Microbiology, 51: 51-58.

Franz, C. M., Muscholl-Silberhorn, A. B., Yousif, N. M. K., Vancanneyt, M., Swings, J., and Holzapfel, W. H. 2001. Incidence of virulence factors and antibiotic resistance among Enterococci isolated from food. Applied Environmental Microbiology, 67: 43854389.

Gevers, D., Danielson, M., Huys, G., and Swings, J. 2003. Molecular characterization of tet (M) genes in Lactobacillus isolates from different types of fermented dry sausage. Applied Environmental Microbiology, 69: 12701275.

Hamilton-Miller, J. M., and Shah, S. 1998. Vancomycin susceptibility as an aid to the identification of lactobacilli. Letters of Applied Microbiology, 26: 153-115.

Herra, C. M., Cafferkey, M. T., and Keane, C. T. 1995. The in vitro susceptibilities of vaginal lactobacilli to four broadspectrum antibiotics, as determined by 
the agar dilution and E-test methods. Journal of Antimicrobial and Chemotherapy, 35: 775-783.

Herreros, M. A., Sandoval, H., Gonzalezn, L., Castro, J. M., Fresno, J. M., and Tornadijo, M. E. 2005. Antimicrobial activity and antibiotic resistance of lactic acid bacteria isolated from armada cheese (a Spanish goats' milk cheese). Food Microbiology, 22: 455459.

Hleba, L., Kacaniova, M., Lejova, J., and Pochop, J. 2011. Antibiotic resistance of Enterobacteriaceae species associated with faecal bacterial cenosis of ducks. Journal of Animal Sciences and Biotechnology, 44: 408-414.

Honi, U., Sabrin, F., Islam, T., Islam, E., Billah, M., and Islam, K. D. 2013. Enzymatic activity and antibiotic resistance profile of Lactobacillus paracasei ssp. paracasei-1 isolated from regional yogurts of Bangladesh. Journal of Microbiology Biotechnology and Food Science, 3: 235-239.

Hummel, A. S., Hertel, C., Holzapfel, W. H., and Franz, C. M. 2007. Antibiotic resistances of starter and probiotic strains of lactic acid bacteria. Applied Environmental Microbiology, 73: 730739.

Huovinen, P. (1987). Trimethoprim resistance. Antimicrobial Agents and Chemotherapy, 31, 1451-1456.

Kastner, S., Perreten, V., Bleuler, H., Hugenschmidt, G., Lacroix, C., and Meile, L. 2006. Antibiotic susceptibility patterns and resistance genes of starter cultures and probiotic bacteria used in food. Systematic and Applied Microbiology, 29: 145-155.

Katla, A. K., Kruse, H., Johnsen, G., and Herikstad, H. 2001. Antimicrobial susceptibility of starter culture bacteria used in Norwegian dairy products.
International Journal of Food Microbiology, 67: 147-152.

Kim, K. S., Morrison, J. O., and Bayer, A. S. 1982. Deficient autolytic enzyme activity in antibiotic tolerant lactobacilli. Infection and Immunity, 36: $582-585$.

Klare, I., Konstabel, C., Müller-Bertling, S., Reissbrodt, R., Huys, G., Vancanneyt, M., Swings, J., Goossens, H., and Witte, W. 2005. Evaluation of new broth media for microdilution antibiotic susceptibility testing of lactobacilli, pediococci, lactococci, and bifidobacteria. Applied Environmental Microbiology, 71: 8982-8986.

Klare, I., Konstabel, C., Werner, G., Huys, G., Vankerckhoven, V., and Kahlmeter, G. 2007. Antimicrobial susceptibilities of Lactobacillus, Pediococcus and Lactococcus human isolates and cultures intended for probiotic or nutritional use. Journal of Antimicrobial Chemothereapy, 59: 900-912.

Klein, G., Hallmann, C., Casas, I. A., Abad, J., Louwers, J., and Reuter, G. 2000. Exclusion of van $A$, van $B$ and van $C$ type glycopeptide resistance in strains of Lactobacillus reuteri and Lactobacillus rhamnosus used as probiotics by polymerase chain reaction and hybridization methods. Journal of Applied Microbiology, 89: 815-824.

Korhonen, J. M., Danielsen, M., Mayo, B., Egervärn, M., Axelsson, L., Huys, G., and Von Wright A. 2008. Antimicrobial susceptibility and proposed microbiological cut-off values of lactobacilli by phenotypic determination. International Journal of Probiotics and Prebiotics, 3: 257-268.

Kushiro, A., Chervaux, C., Cools-Portier, S., Perony, A., Legrain-Raspaud, S., Obis, D., Onoue, M., and van der Moer, A. 2009. Antimicrobial susceptibility testing of lactic acid bacteria and bifidobacteria by broth micro-dilution test 
and E-test. International Journal of Food Microbiology, 132: 54-58.

Lapsiri, W., Nitisinprasert, S., and Wanchaitanawong, P. 2011. Lactobacillus plantarum strains from fermented vegetables as potential probiotics. Kasetsart Journal, 45: 1071-1082.

Lavanya, B., Sowmiya, S., Balaji, S., and Muthuvelan, B. 2011. Plasmid profiling and curing of Lactobacillus strains isolated from fermented milk for probiotic applications. Advance Journal Food Science Technology, 3: 95-101.

Lira, W. M., Macedo, C., and Marin, J. M. 2004. The incidence of Shiga toxinproducing Escherichia coli in cattle with mastitis in Brazil. Journal of Applied Microbiology, 97: 861-866.

Liu, C., Zhang, Z. Y., Dong, K., Yuan, J. P., and Guop, X. K. 2009. Antibiotic resistance of probiotic strains of lactic acid bacteria isolated from marketed foods and drugs. Biomedical and Environmental Sciences, 22: 401-412.

Mater, D. D., Langella, P., Corthier, G., and Flores, M. J. 2008. A probiotic Lactobacillus strain can acquire vancomycin resistance during digestive transit in mice. Journal of Molecular Microbiology Biotechnology, 14: 123127.

Mayrhofer, S., vanHoek, A. H. A. M., Mair, C., Huys, G., Arts, H. J. M., Kneifel, W., and Domig, K. J. (2010). Antibiotic susceptibility of members of the Lactobacillus acidophilus group using broth microdilution and molecular identification of their resistance determinants. International Journal Food Microbiology, 144: 81-87.

Nawaz, M., Wang, J., Zhou, A., Ma, C., Wu, X., Moore, J. E., Millar, B. C., and Xu, J. 2011. Characterization and transfer of antibiotic resistance in lactic acid bacteria from fermented food products. Current Microbiology, 62: 1081-1089.

Olukoya, D. K., Ebigwei, S. I., Adebawo, O. O., and Osiyemi, F. O. 1993. Plasmid profiles and antibiotic susceptibility patterns of
Lactobacillus isolated from fermented foods in Nigeria. Food Microbiology, 10: 279-285.

Pérez-Pulido, R., Benomar, N., Abriouel, H., Lucas, López, R., Martínez-Cañamero, M., and Gálvez, A. 2005. Microbiological study of lactic acid fermentation of caper berries by molecular and culturedependent methods. Applied Environmental Microbiology, 71: 78727879 .

Phillips, I., Andrews, J. M., Bridson, E., Cooke, E. M., Spencer, R. C., Holt, H. A., Wise, R., Bint, A. J., Brown, D. F. J., Greenwood, D., King, A., and Williams, R. J. 1991. A Guide to Sensitivity Testing. Report of the Working Party on Antibiotic Sensitivity Testing of the British Society for Antimicrobial Chemotherapy, 27: 1-50.

Picozzi, C., Foschino, R., Heuvelink, A., and Beumer, R. 2005. Phenotypic and genotypic characterization of sorbitolnegative or slow-fermenting (suspected O157) Escherichia coli isolated from milk samples in Lombardy region. Letters in Applied Microbiology, 40: 491-496.

Putman, M. H., van, Veen, Degener, J. E., and Lonnigs, W. N. 2001. The Lactococcal secondary multidrug transporter LmrP confers resistance to lincosamides, macrolides, streptogrmins and tetracyclines. Microbiology, 147: 28732880.

Rosander, A., Connolly, E., and Roos, S. 2008. Removal of antibiotic resistance genecarrying plasmids from Lactobacillus reuteri ATCC 55730 and characterization of the resulting daughter strain, $L$. reuteri DSM 17938. Applied Environmental Microbiology, 74: 6032-6040.

Rozila, A., Rajandara, P., Lani, M. N., Siti, Hasmah. M., Ezni, Suryani. I., and Ragupathy, R. 2012. Antibiotic resistant of Lactobacillus isolated from dairy products. UMT 11th International Annual Symposium on Sustainability Science and Management 09th-11th July 2012. Terengganu: Malaysia. 
Sharma, P., Tomar, S. K., Goswami, P., Sangwan, V., and Singh, R. 2014. Antibiotic resistance among commercially available probiotics. Food Research International, 57: 176-195.

Sharma, P., Tomar, S.K., Sangwan, V., Goswami, P., and Singh, R. (2015). Antibiotic resistance of Lactobacillus sp. isolated from commercial probiotic preparations. Journal of Food Safety, 36: 38-51.

Soomro, A. H., and Masud, T. 2012. Probiotic characteristics of Lactobacillus spp. isolated from fermented milk product Dahi. Food Science and Technology Research, 18: 91-98.

Tannock, G. W., Luchansky, J. B., Miller, L., Connell, H., Thodeandersen, S., and Mercer, A. A. 1994. Molecular characterization of a plasmid borne (pGT633) erythromycin resistance determinant (ermGT) from Lactobacillus reuteri. Plasmid, 31: 60-71.

Temmerman, R., Pot, B., Huys, G., and Swings, J. 2003. Identification and antibiotic susceptibility of bacterial isolates from probiotic products. International Journal of Food Microbiology, 81, 1-10.

Teuber, M., Meile, L., and Schwarz, F. 1999. Acquired antibiotic resistance in lactic acid bacteria from food. Antonie Van Leeuwenhoek, 76: 115-137.

Thumu, S. C., and Halami, P. M. 2012. Presence of erythromycin and tetracycline resistance genes in lactic acid bacteria from fermented foods of Indian origin. Antonie Van Leeuwenhoek, 102: 541-551.

Turnidge, J. D., and Bell, J. M. 2005. Antimicrobial susceptibility on solid media. In A. Lorian (Ed.), Antibiotics in laboratory medicine (pp. 8-60). Baltimore, USA: Lippincott Williams and Wilkins.

Wong, A., Ngu, Y. H., Dan, L.A., Ooi, A., and Lim, R. L. H. 2015. Detection of antibiotic resistance in probiotics of dietary supplements. The Journal of Nutrition, 14: 95-102.

Yüksekdağ, Z. N., and Beyatli, Y. 2008. Antimicrobial activity and antibiotic susceptibility of some lactic acid bacteria isolated different dairy products. Archiv für Lebensmittel Hygiene, 59: 216-220.

Zarazaga, M., Sainz, Y., Portillo, A., Tenorio, C., Ruiz-Larrara, R. F., Campo, R., Baquero, O. F., and Torres, C. 1999. In vitro activities of ketolide HMR3647, macrolides, and other antibiotics against Lactobacillus, Leuconostoc and Pediococcus isolates. Antimicrobial Agents Chemotherapy, 43: 3039- 3041.

Zdolec, N., Filipovic, I., Cvrtila, Fleck. Z., Maric, A., Jankuloski, D., and Kozacinski, L. 2011. Antimicrobial susceptibility of lactic acid bacteria isolated from fermented sausages and raw cheese. Veterinarski Arhiv, 81: 133-141.

Zhang, Y. 2007. Mechanisms of antibiotic resistance in the microbial world. Clinical Pharmacology and Therapeutics, 82: 595-600.

Zhou, N., Zhang, J. X., Fan, M. T., Wang, J., Guo, G., and Wei, X. Y. 2012. Antibiotic resistance of lactic acid bacteria isolated from Chinese yogurts. Journal of Dairy Science, 95: 4775-4783.

\section{How to cite this article:}

Poonam Sharma, Santosh Anand, Sudhir Kumar Tomar and Pawas Goswami. 2018. Antibiotic Susceptibility of Lactobacillus sp. Isolated from Commercial Probiotic Products by E-Test Strip Method. Int.J.Curr.Microbiol.App.Sci. 7(04): 3499-3517. doi: https://doi.org/10.20546/ijcmas.2018.704.396 